\title{
Roughness Dependence of the Casimir Force between Fractal Surfaces
}

\author{
Kosuke Goto, Kozo Mochiji, Kousuke Moritani, and Norio Inui* \\ Graduate School of Engineering, University of Hyogo, \\ 2167 Shosha, Himeji, Hyogo 671-2280, Japan \\ (Received 7 May 2014; Accepted 17 June 2014; Published 5 July 2014)
}

\begin{abstract}
We study the surface-roughness dependence of the Casimir force between fractal surfaces, which is represented by the Weierstrass-Mandelbrot function. When compared the Casimir force in the case of smooth surfaces, the Casimir force between fractal surfaces rapidly increases as the separation distance decreases. We express this deviation as a simple analytical function that is composed of three parameters including fractal dimension, and compare it with the deviation obtained from Monte Carlo simulations.

[DOI: $10.1380 /$ ejssnt.2014.313]
\end{abstract}

Keywords: Quantum effects; Atomic force microscopy; Metallic surfaces; Computer simulations; Surface structure, morphology, roughness, and topography

\section{INTRODUCTION}

Surface forces play a very important role in surface interactions, such as adhesion, stiction, and wetting [1]. The Casimir force is a major contributing factor in surface forces when surfaces are electrically neutral, and the separation is much larger than the atomic scale [2-5]. The Casimir force arising from a change in zero-point vacuum fluctuations very rapidly increases as the separation between the objects decreases. Thus, the Casimir force has been a topic of great interest in the area of microelectromechanical systems (MEMS) for the past decade [6$8]$, and the rapid theoretical progress with respect to the Casimir effect enables us to predict the Casimir force between many materials [9]. However, many difficulties still remain. The major difficulty arises from the fact that the Casimir force strongly depends on shape; as a result, we cannot exactly calculate the Casimir force between rough surfaces yet.

The Casimir force nonlinearly increases as the separation distance between objects decreases. Thus, the influence of surface roughness on the Casimir force becomes significantly large, especially in the case of small separations. Several methods of computing the Casimir force between rough surfaces have been already proposed, and the influence of roughness on the Casimir force has also been studied [10-12]. However, since there are various surface shapes that exist in real materials, it is difficult to determine the roughness dependence of the Casimir force comprehensively. In this study, we restrict our attention to the fractal surface described by the WeierstrassMandelbrot (WM) function [13]; however, we give simple analytical results for the roughness dependence of the Casimir force.

The WM function is defined by the superposition of trigonometric functions, whose power spectrum exhibits a power-law decay and the phase is random. This function has been used to represent engineering surfaces produced by random processes, in which self-similarity is often observed at the surface [14]. The knowledge of the self-affine

*Corresponding author: inui@eng.u-hyogo.ac.jp

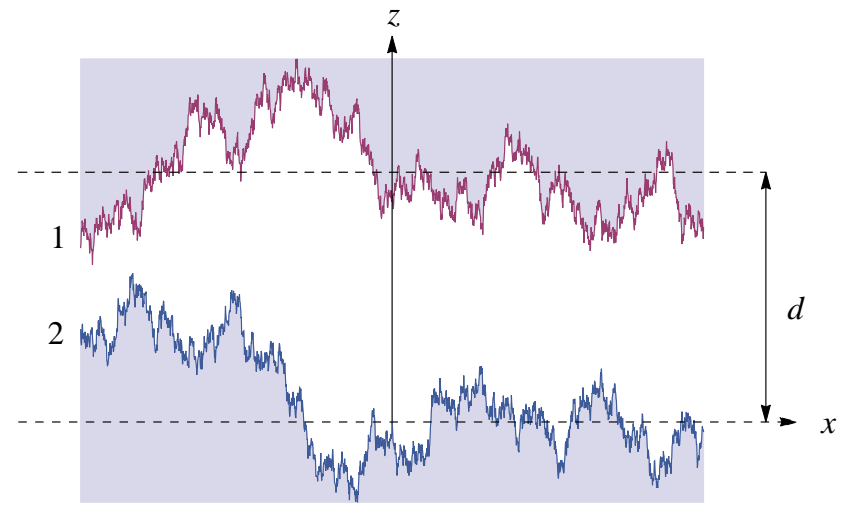

FIG. 1: Two parallel plates with WM functions.

structure of the surface is important factor for understanding roughness studies, and the self-affine structure of the surface has been measured by various methods. For example, Palasantzas measured the height-height correlation of a silver film deposited on a polished quartz crystal by scanning tunneling microscopy and compared it to an analytical form [15]. Furthermore, fractal surface is important to surface modification technology. For example, it has been shown that fractal surfaces can be super water repellent [16]. We use the WM function to investigate the roughness dependence of the Casimir force because this function is characterized by few parameters. If massive height profile data sets are used, a good approximation of the correction to the Casimir force may be obtained, but this is not the aim of this study. We aim to understand the roughness dependence of the Casimir force between fractal surfaces by as few parameters as possible.

This paper is organized as follows. In Sec. II, we introduce the WM function and calculate the height-height correlation. In Sec. III, using Monte Carlo simulations, the statistical properties of the roughness profile are expressed by a Gaussian function, and the standard deviation is approximated by a simple analytical form. In Sec. IV, the roughness dependence of the Casimir force between two independent fractal surfaces is considered. We give a simple analytical form to the ratio of the Casimir 


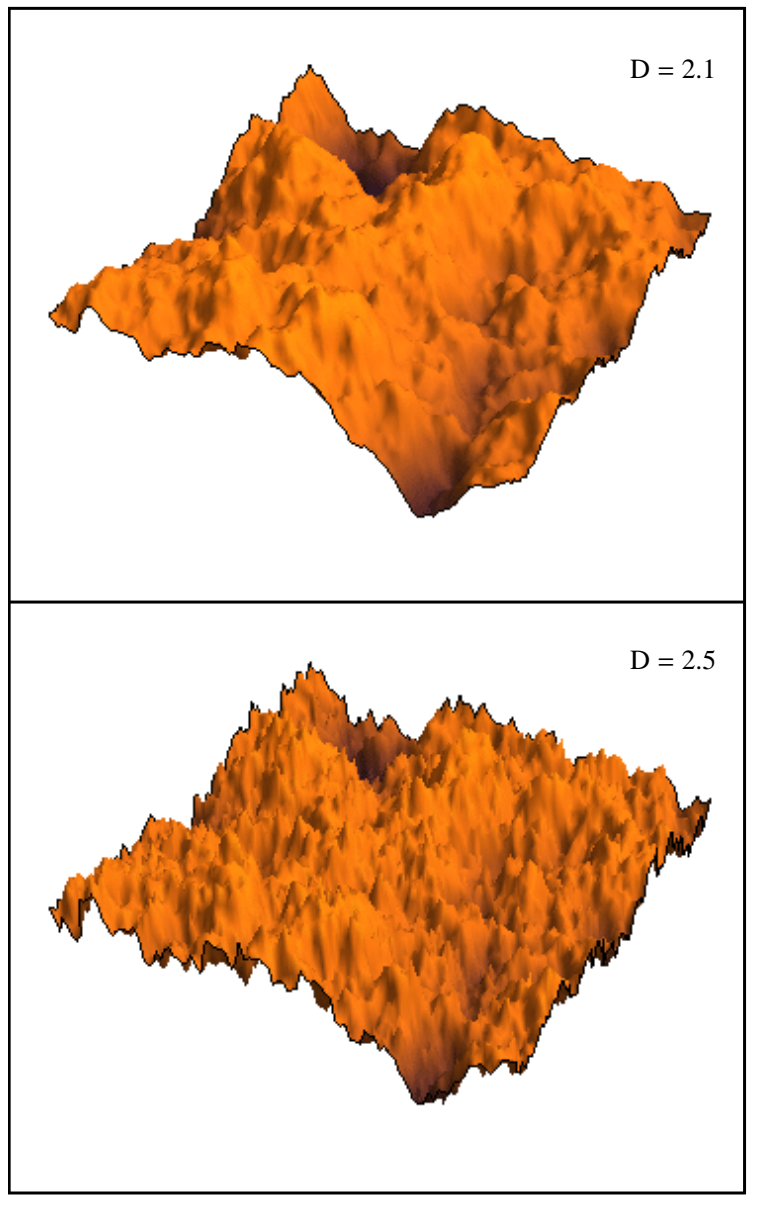

FIG. 2: WM functions with $D=2.1$ and 2.5 generated using the same set of random phases. force between fractal surfaces to that between flat surfaces at the same separation distance. Finally, we present our conclusions and discuss the limitations of the approximation form of the Casimir force between fractal surfaces.

\section{CORRELATION FUNCTION OF THE WEIERSTRASS-MANDELBROT FUNCTION}

Mandelbrot found that self-similar patterns are observed in various natural phenomena and termed the shape with self-similar patterns as fractal [17]. The fractal is very useful for understanding surface roughness because roughness measurements of various surfaces have shown that their topographies are fractal. The fractal surface observed in nature is very complicated, and it is impossible to be described by smooth functions. However, Mandelbrot showed that the function derived by Weierstrass, which is a superimposition of infinite trigonometric functions, is fractal and although continuous everywhere is differentiable nowhere. In this study, we call this function the WM function. The WM function is initially defined by a function of a single variable and is generalized to that of multiple variables by Ausloos and Berman [18].

We consider two parallel plates with side length $L$ and surface roughness described by the generalized WM function, as shown in Fig. 1. We denote the upper and lower surface as $z_{1}(x, y)$ and $z_{2}(x, y)+d$, where $d$ is the mean separation between the surfaces. The generalized WM function, which is characterized by three parameters $A$, $\gamma$, and fractal dimension $D(2<D<3)$, is defined by

$$
z(x, y)=\frac{A}{\sqrt{M}} \sum_{m=1}^{M} \sum_{n=0}^{N} \gamma^{(D-3) n}\left\{\cos \phi_{n, m}-\cos \left[a_{n, m}(x, y) \sqrt{\left(\frac{x}{L}\right)^{2}+\left(\frac{y}{L}\right)^{2}}+\phi_{n, m}\right]\right\}
$$

where $\phi_{n, m}$ is a random phase taken from a uniform distribution between 0 and $2 \pi$, and $a_{n, m}(x, y)$ is defined by

$$
a_{n, m}(x, y)=2 \pi \gamma^{n} \cos \left[\tan ^{-1}\left(\frac{y}{x}\right)-\frac{\pi m}{M}\right] .
$$

Strictly speaking, the above function is not a perfect fractal if $N$ and $M$ are finite. However, a minimum length exists in real surfaces, and the properties of the fractal are observed at larger scales than the minimum length if $N$ and $M$ are large. Figure 2 shows examples of the WM function with $\gamma=1.5, N=17$, and $M=10$ for $D=2.1$ and 2.5 , in which the phase $\phi_{n, m}$ is unchanged for each $n$ and $m$. We assume that $M$ is odd in this study for simplicity.

We consider the statistical properties of the WM function. We define mean of the roughness by

$$
\bar{z}=\frac{1}{L^{2}} \int_{0}^{L} \int_{0}^{L} z(x, y) d x d y
$$

Since the WM function includes a random phase, the infinite different surfaces are generated depending on a random phase. Thus, we consider an ensemble average of a statistic of the roughness $X$ over all possible surfaces and denote it by $\langle X\rangle$. For example, the ensemble average of the mean roughness is denoted by $\langle\bar{z}\rangle$, and it is zero. One of the important statistics is the height-height correlation function of the WM function along the $x$ axis:

$$
R(\tau)=\frac{1}{L} \int_{0}^{L} z(x) z(x+\tau) d x
$$

The average height-height correlation function of $\tau$ is given by 


$$
\begin{aligned}
\langle R(\tau)\rangle & =\frac{A^{2}}{2}\left[\frac{1-\left(\gamma^{2 D-6}\right)^{N}}{1-\gamma^{2 D-6}}+\sum_{m=1}^{M} \sum_{n=0}^{N} \gamma^{2(D-3) n}\left(f_{n, m}^{(1)}(\tau)+f_{n, m}^{(2)}(\tau)\right)\right], \\
f_{n, m}^{(1)}(\tau) & =\frac{2 \sin \left(\frac{a_{n, m}}{2}\right)}{a_{n, m}}\left[\cos \left(\frac{a_{n, m}}{2}\right)+\cos \left\{a_{n, m}\left(\frac{1}{2}+\frac{\tau}{L}\right)\right\}\right], \\
f_{n, m}^{(2)}(\tau) & =\cos \left(a_{n, m} \frac{\tau}{L}\right) .
\end{aligned}
$$

The derivation of this equation is given in Appendix A.

For large $M$, the variable $m / M$ can be approximated as a continuous variable. Thus, the summation over $m$ can be replaced with an integral, and the following formula is obtained

$$
\left\langle R_{\infty}(\tau)\right\rangle=\frac{A^{2}}{2}\left[\frac{1-\left(\gamma^{2 D-6}\right)^{N}}{1-\gamma^{2 D-6}}+\sum_{n=0}^{N} \gamma^{2(D-3) n} g_{n}(\gamma, \tau)\right],
$$

where $g_{n}(\gamma, \tau)$ can be expressed by several special functions. The derivation of this equation and definition of $g_{n}(\gamma, \tau)$ are given in Appendix A. Here we note that $\langle R(\tau)\rangle$ can be written as a function of the ratio of $\tau$ and $L$.

Figure 3 shows the comparison of the height-height correlation function given in (5) for $N=10, M=11$, $D=2.1, \gamma=1.5$, and $A / \sqrt{M}=1$ with that by Monte Carlo simulations with $10^{5}$ surfaces. The agreement between the two methods is remarkably good. In the limit of $M \rightarrow \infty$, the height-height correlation function converges to $\left\langle R_{\infty}(\tau)\right\rangle$, shown by a solid line in Fig. 3 .

\section{DISTRIBUTION FUNCTION OF ROUGHNESS}

We consider the distribution function of roughness $P(\zeta)$, that is the probability of the measuring height between $\zeta$ and $\zeta+d \zeta$ in the area $L \times L$. We show $P(\zeta)$ for $N=10, M=11, D=2.1, \gamma=1.5$, and $A / \sqrt{M}=1 \mathrm{ob}-$

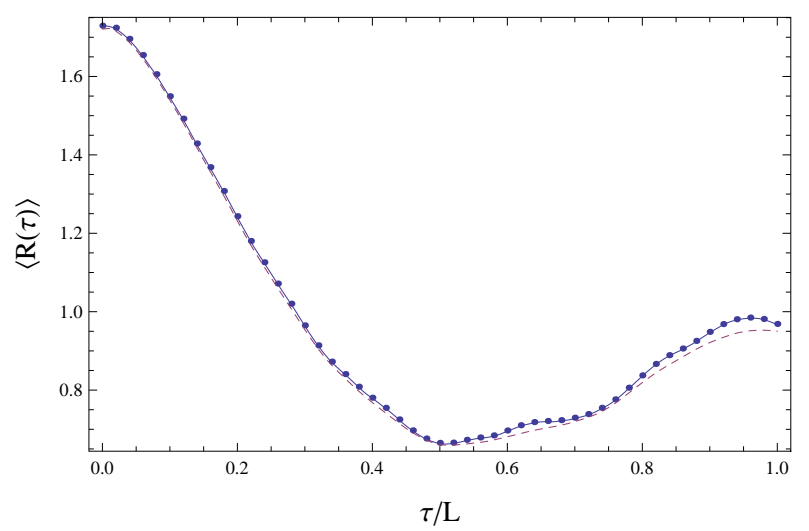

FIG. 3: Plot of the averaged height-height correlation function with $D=2.1$ and $\gamma=1.5$. Solid circles and the solid line show the values obtained by Monte Carlo simulations and the formula in (7), respectively. The dashed line shows the average height-height correlation function in the limit of $M \rightarrow \infty$. tained by Monte Carlo simulations with $10^{5}$ surfaces (solid circles) and Gaussian fitting (solid line), whose variance is 2.5 as an example in Fig. 4. The distribution function of roughness seems to be well approximated by the Gaussian function.

Since the mean of the surface roughness is zero, the distribution function is mainly determined by the variance of surface roughness. The variance in the limit of $M, N \rightarrow$ $\infty$ is the height-height correlation function at $\tau=0$ (see Appendix A):

$$
\begin{aligned}
\sigma^{2} & =\left\langle R_{c}(0)\right\rangle \\
& =\frac{A^{2}}{1-\gamma^{2 D-6}}(1+\delta(\gamma, D))
\end{aligned}
$$

where

$$
\delta(\gamma, D) \equiv\left(1-\gamma^{2 D-6}\right) \sum_{n=0}^{\infty} \gamma^{2(D-3) n} g_{1}(\gamma)
$$

Figure 5 shows $\delta(\gamma, D)$ as a function of the fractal dimension $D$ for $\gamma=1.5$ and 2.0. The absolute value of $\delta(\gamma, D)$

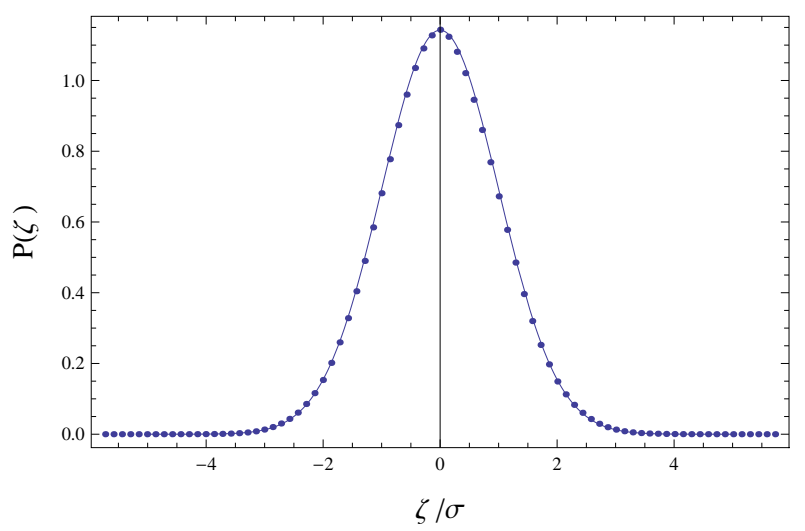

FIG. 4: Distribution function of surface roughness described by the WM function with $D=2.1$ and $\gamma=1.5$. Solid circles and the dashed line show the value obtained by Monte Carlo simulations and the Gaussian function, whose variance is given by (A20). 


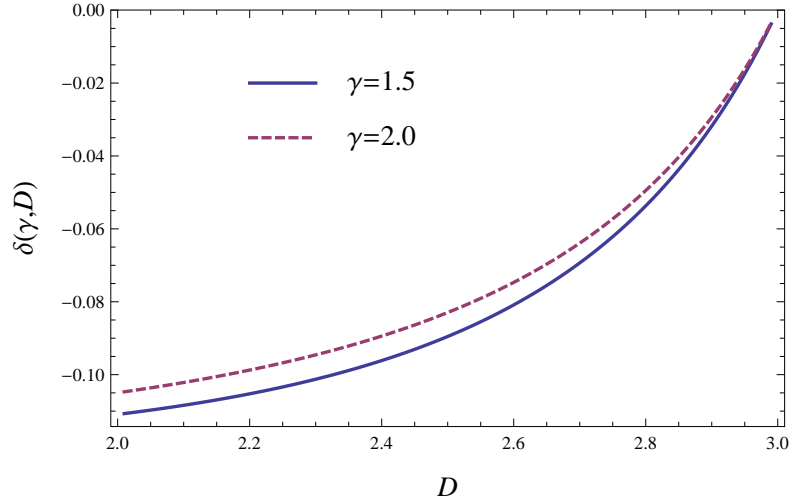

FIG. 5: Plot of $\delta(\gamma, D)$ for $\gamma=1.5$ and 2.0, which concerns a correction term of the variance of roughness distribution. If $\delta(\gamma, D)=0$, the variance is exactly expressed by $A^{2} /(1-$ $\left.\gamma^{2 D-6}\right)$.

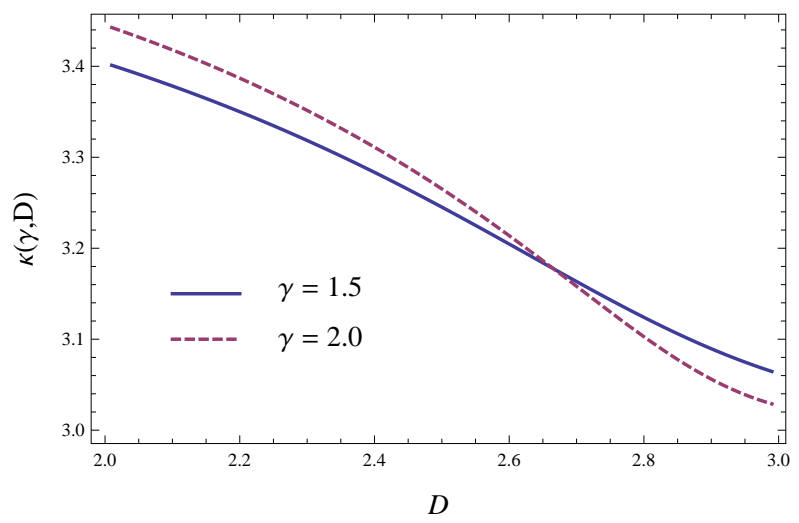

FIG. 6: Plot of the kurtosis of the roughness distribution for $\gamma=1.5$ and 2.0. The kurtosis of the Gaussian function is 3. The roughness distribution is well approximated by the Gaussian function as the fractal dimension increases.

increases as $D$ decreases. However, $\delta(\gamma, D)$ is limited to several percent of the variance except for near $D=2$. Thus, we can approximately estimate the variance of $P(\zeta)$ as $A^{2} /\left(1-\gamma^{2 D-6}\right)$.

To verify that the distribution function can be approximated by the Gaussian function, we calculate the kurtosis of the surface roughness $\kappa(\gamma, D)$, defined by

$$
\kappa(\gamma, D)=\frac{m_{4}}{\sigma^{4}}
$$

where $m_{4}$ is the fourth moment of the distribution function $P(\zeta)$ (see Appendix B). Since the kurtosis of the Gaussian function is exactly 3 independent of the variance, the deviation of the kurtosis from 3 represents the degree of the Gaussian fitting approximation. Figure 6 shows $\kappa(\gamma, D)$ with $N=10$ and $M=11$ as a function of the fractal dimension for $\gamma=1.5$ and 2.0. The kurtosis of the roughness distribution shown in Fig. 4 is 3.38. If the kurtosis is less than this value, then the roughness distribution function is more fitted to the Gaussian function. We remark that the kurtosis is independent of the amplitude $A$.

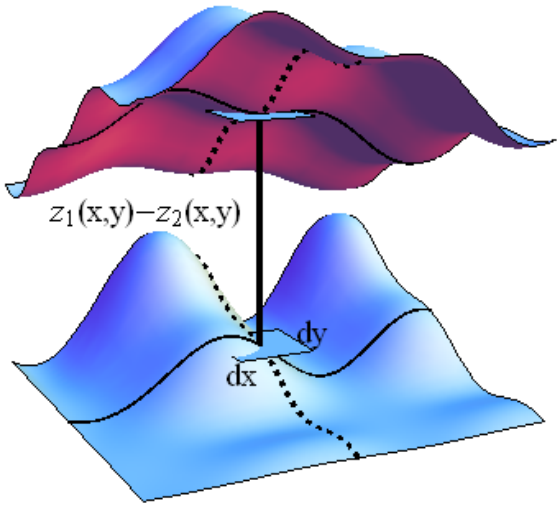

FIG. 7: Illustration of the proximity force approximation for the Casimir energy between rough surfaces. The Casimir energy density is approximated by that between parallel plates with a separation gap $z_{1}-z_{2}$. PFA is only valid when the roughness is much smaller than the separation distance.

\section{CORRECTION OF SURFACE ROUGHNESS TO THE CASIMIR FORCE}

We start to consider the Casimir force between perfectly conductive plates. If their surface is smooth and the separation distance between plates is $d$, the Casimir energy $E_{0}(d)$ and force $F_{0}(d)$ per area are given by

$$
\begin{aligned}
E_{0}(d) & =-\frac{\pi^{2} \hbar c}{720 d^{3}}, \\
F_{0}(d) & =-\left.\frac{\partial E(z)}{d z}\right|_{z=d}, \\
& =-\frac{\pi^{2} \hbar c}{240 d^{4}}
\end{aligned}
$$

If the surface is smooth but the conductivity of the plate considered above is not infinity, the Casimir energy depends on the dielectric permittivity of plates. When the dielectric permittivity of plates at angular frequency $\omega$ can be described by the plasma model $\epsilon(\omega)=1-\omega_{p}^{2} / \omega^{2}$, the Casimir energy is

$$
E_{p}(d)=\frac{\pi^{2} \hbar c}{720 d^{3}}\left[1-4 \frac{\delta}{d}+\frac{72}{5}\left(\frac{\delta}{d}\right)^{2}+\mathcal{O}\left(\frac{\delta}{d}\right)^{3}\right]
$$

where $\delta$ is the skin depth $c / \omega_{p}$ [19]. To focus our attention on the roughness dependence of the Casimir energy, we consider only the separation distance much larger than the skin depth. In addition, we assume the variance of the surface is much smaller than the separation distance.

If the surface is not smooth, we hardly calculate the exact Casimir force. Here, we introduce the proximity force approximate formula (PFA). In PFA, as shown in Fig. 7, the contribution of the Casimir energy in a rectangular parallelepiped with an area of the base $d x d y$ and a height $z_{1}-z_{2}$ to the Casimir energy between rough surfaces is approximated by the Casimir energy between perfectly conductive parallel plate $\pi^{2} \hbar c /\left[720\left(z_{1}-z_{2}\right)^{3}\right] d x d y$. As a result, the Casimir energy between rough surfaces of side 
length $L \times L$ is approximated by

$$
E(d)=-\frac{\pi^{2} \hbar c}{720 L^{2}} \int_{0}^{L} d x \int_{0}^{L} d y \frac{1}{\left(z_{1}(x, y)-z_{2}(x, y)\right)^{3}}
$$

where $z_{1}(x, y)-z_{2}(x, y)$ is the separation distance between the plate at the point with coordinates $(x, y)$.

Let us calculate the Casimir energy between plates whose roughness can be described by the WM function. Since the roughness distribution of the WM function is approximately expressed by the Gaussian function, the Casimir force between the WM surfaces is approximately given by

$$
F(d)=-\frac{\pi^{2} \hbar c}{240} \int_{z_{\min }}^{\infty} \frac{1}{(d+z)^{4}} \frac{e^{-\frac{z^{2}}{2 \sigma^{2}}}}{\sqrt{2 \pi \sigma^{2}}} d z
$$

where $\sigma$ is the standard deviation of the separation distance, and $z_{\min }$ is the cut-off deviation. If the surface is perfectly flat, the separation can decreases unlimitedly. However, the rough surfaces come into contact at a finite $d$ by decreasing the separation. This separation distance depends on the randomness used in the WM function. Thus, the above formulation of the Casimir force is available for much larger sedations than $\sigma$. The probability of roughness larger than $\sigma$ is small but not zero; we need to introduce the cut-off for deviation. If we can set a cutoff deviation satisfying the condition $-d \ll z_{\text {min }} \ll \delta$, we may obtain a good approximation value for the Casimir force. We express the Eq. (18) in a simple form using the Taylor expansion. The integrand in Eq. (18) is expressed as

$$
\begin{gathered}
f(z)=\frac{1}{d^{4}} \sum_{n=0}^{\infty} \frac{(-1)^{n}}{6}(n+1)(n+2)(n+3)\left(\frac{z}{d}\right)^{n} \frac{e^{-\frac{z^{2}}{2 \sigma^{2}}}}{\sqrt{2 \pi \sigma^{2}}}, \\
\text { for } \quad z>-d .
\end{gathered}
$$

Although this series expansion converges only for $z>-d$, the integration of each term with respect to $z$ from $-\infty$ to $\infty$ converges, and we can express it in an analytical form by using the following formula:

$$
\int_{-\infty}^{\infty} z^{n} e^{-a^{2} z^{2}} d z= \begin{cases}0, & n=\text { odd } \\ \frac{\Gamma\left(\frac{n+1}{2}\right)}{a^{n+1}}, & n=\text { even }\end{cases}
$$

where $\Gamma(x)$ denotes the gamma function. If the contribution of the integration on $\left(-\infty, z_{\min }\right]$ is small with respect to the Casimir force, the Casimir force is approximately given by

$$
F(d) \approx \frac{\pi^{2} \hbar c}{240 d^{4}} \sum_{n=0}^{\infty} C_{n}\left(\frac{\sigma}{d}\right)^{2 n}
$$

where

$$
C_{n}=\frac{1}{3}(n+1)\left(4(n+1)^{2}-1\right)(2 n-1) ! ! .
$$

Here, $(2 n-1) !$ ! is defined as $1 \times 3 \times 5 \times \cdots \times(2 n-$ 1 ). Consequently, the ratio of $F(d)$ to the Casimir force

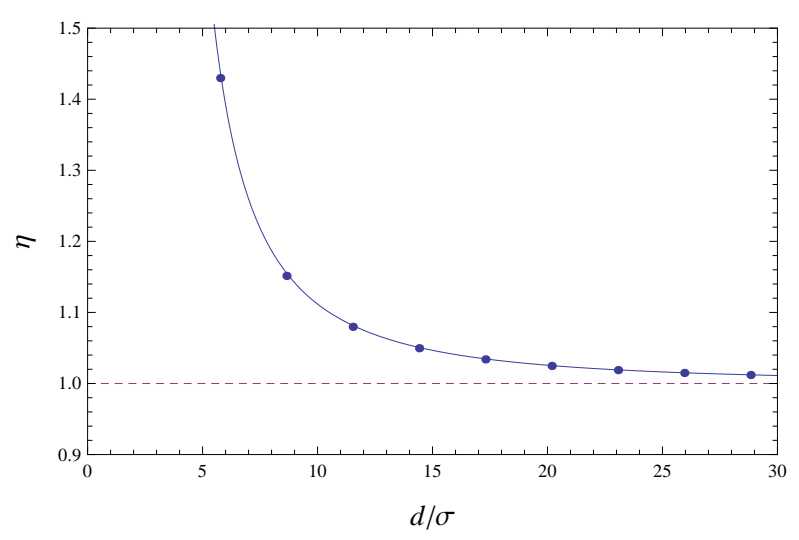

FIG. 8: Ratio of the Casimir force between rough surfaces to that between flat surfaces. Solid circles obtained by Monte Carlo simulation agree well with the approximate function given by Eq. (25). The ratio converges to 1, which is indicated by a dashed line if the separation is much larger than the standard deviation of the roughness.

between the smooth surface is given by

$$
\begin{aligned}
\eta(d, \sigma) & \equiv \frac{F(d)}{F_{0}(d)} \\
& \approx\left\{1+10\left(\frac{\sigma}{d}\right)^{2}+105\left(\frac{\sigma}{d}\right)^{4}+1260\left(\frac{\sigma}{d}\right)^{6}+\ldots\right\}
\end{aligned}
$$

This equation coincides with a special case of the correction formula obtained by Bordag et al. [4, 20]. Using Padé approximation, the roughness correction of the Casimir force can be expressed as a function of the ratio $\xi \equiv \sigma / d$ :

$$
\eta(d, \sigma) \approx \frac{1-\frac{1}{2} \xi^{2}}{1-\frac{21}{2} \xi^{2}}
$$

This approximation is available for $\xi \ll \frac{\sqrt{2}}{21}$.

Figure 8 shows the comparison of the roughness correction $\eta$ for the WM surface with $\sigma_{1}=\sigma_{2}=1.32$ obtained by the Monte Carlo method, which is averaged over $10^{3}$ surfaces, with the value obtained by Eq. (25). The deviation from the Casimir force between the flat surfaces rapidly increases as the surface separation decreases and it can be expressed by a very simple rational function. We have already shown the ensemble average of the standard deviation of the WM surface by three parameters A, $\gamma$, and $D$. The variance of the separation distance between the WM surface with variance $\sigma_{1}^{2}$ and $\sigma_{2}^{2}$ is $\sigma_{1}^{2}+\sigma_{2}^{2}$. As a result, by combining Eqs. (11) and (25), we can estimate the deviation of the Casimir force between the WM surfaces if three parameters are known.

\section{CONCLUSIONS}

We considered the influence of roughness on the Casimir force. The strength of the Casimir force rapidly increases as the separation distance decreases. Thus, the influence of roughness is considerably important at small separations. Since the Casimir force at small separations often 
causes adhesion between MEMS parts, surface roughness is essential factor for designing MEMS. However, even if we preciously know surface topography, it is difficult to accurately calculate the Casimir force because the Casimir force is not expressed by the summation of the force between two atoms, and we need to obtain the electromagnetic field satisfying the appropriate boundary conditions at rough surfaces. In addition, the surface topography is individually changed, and it is impossible to measure each surface of MEMS parts. Thus, it is important to know the statistical properties of the Casimir force between rough surfaces.

We focused our attention on fractal surfaces because engineering surfaces are often fractal. Many frequency components are included in the spectrum of fractal surfaces, and its power spectral density exhibits a power-law decay. Fractal curves are although continuous everywhere are differentiable nowhere. The WM function is a well-known function that exhibits these properties and has been used to study the contact between rough surfaces. We showed that the distribution of roughness of the WM function is approximated as a Gaussian function, and its standard deviation is expressed by three parameters. The correction of the Casimir force between the surfaces characterized by the WM function to that between flat surfaces was expressed as a function of the ratio of the separation distance to its standard deviation. Since the relation between the standard deviation and three parameters was obtained, the correction of the Casimir force can be also considered in the terms of three parameters. For example, for a fixed mean separation-distance, we can show that the strength of the Casimir force increases as the fractal dimension increases.

We restricted our attention to the correction of the Casimir force between perfectly conductive plates. However, the results obtained in this study are easily generalized for real materials. For example, the strength of the Casimir force between metallic plates decreases inversely proportionally to $d^{3}$ for small separations. Accordingly, the correction of the Casimir force can be calculated by changing the exponent $(d+z)$ in Eq. (18) from 4 to 3 .

\section{Acknowledgments}

This research was supported by the Ministry of Education, Culture, Sports, Science and Technology, Grant-inAid for Scientific Research(C), MEXT KAKENHI Grant Number 13240323 and Sanyo Special Steel Cultural Promotion Foundation.

\section{Appendix A: Height-height correlation function}

The derivation of the height-height correlation function along the $x$ axis is as follows. The WM function at $y=0$ is given by

$$
z(x)=\frac{A}{\sqrt{M}} \sum_{m=1}^{M} \sum_{n=0}^{N} \gamma^{(D-3) n}\left\{\cos \phi_{n, m}-\cos \left[a_{n, m}\left(\frac{x}{L}\right)+\phi_{n, m}\right]\right\},
$$

where $a_{n, m}$ is defined by

$$
a_{n, m}=2 \pi \gamma^{n} \cos \left(\frac{\pi m}{M}\right)
$$

Substituting Eq. (A1) into Eq. (4), we have

$$
R(\tau)=\frac{A^{2}}{M L} \int_{0}^{L} \sum_{m_{1}=1}^{M} \sum_{n_{1}=0}^{N} \sum_{m_{2}=1}^{M} \sum_{n_{2}=0}^{N} \zeta_{n_{1}, m_{1}}(x) \zeta_{n_{2}, m_{2}}(x+\tau) d x .
$$

Here $\zeta_{n, m}(x)$ is defined by

$$
\zeta_{n, m}(x)=\gamma^{(D-3) n}\left\{\cos \phi_{n, m}-\cos \left[a_{n, m}\left(\frac{x}{L}\right)+\phi_{n, m}\right]\right\} .
$$

The ensemble average of the height-height correlation function is given by

$$
\begin{aligned}
\langle R(\tau)\rangle & =\left\langle\frac{A^{2}}{M L} \int_{0}^{L} \sum_{m=1}^{M} \sum_{n=0}^{N} \gamma^{2(D-3) n} \sum_{k=1}^{3} f_{n, m}^{(k)}(x) d x\right\rangle \\
f_{n, m}^{(1)}(x) & =\cos ^{2} \phi_{n, m} \\
f_{n, m}^{(2)}(x) & =-\cos \phi_{n, m} \cos \left[a_{n, m}\left(\frac{x}{L}\right)+\phi_{n, m}\right]-\cos \phi_{n, m} \cos \left[a_{n, m}\left(\frac{x+\tau}{L}\right)+\phi_{n, m}\right] \\
f_{n, m}^{(3)}(x) & =\cos \left[a_{n, m}\left(\frac{x}{L}\right)+\phi_{n, m}\right] \cos \left[a_{n, m}\left(\frac{x+\tau}{L}\right)+\phi_{n, m}\right] .
\end{aligned}
$$


Here we used the following equation for any arbitrary function $f\left(n_{1}, m_{1}, n_{2}, m_{2}\right)$ including no random phase:

$$
\left\langle\sum_{m_{1}=1}^{M} \sum_{n_{1}=0}^{N} \sum_{m_{2}=1}^{M} \sum_{n_{2}=0}^{N} \cos \phi_{n_{1}, m_{1}} \cos \phi_{n_{2}, m_{2}} f\left(n_{1}, m_{1}, n_{2}, m_{2}\right)\right\rangle=\frac{1}{2} \sum_{n=1}^{M} \sum_{m=1}^{M} f\left(n_{1}, m_{1}, n_{2}, m_{2}\right) .
$$

The contribution of the term including $f_{n, m}^{(1)}$ to $\langle R(\tau)\rangle$ is given by

$$
\left\langle\frac{A^{2}}{M L} \int_{0}^{L} \sum_{m=1}^{M} \sum_{n=0}^{N} \gamma^{2(D-3) n} f_{n, m}^{(1)} d x\right\rangle=\frac{A^{2}}{2} \sum_{n=0}^{N} \gamma^{2(D-3) n}=\frac{A^{2}}{2} \frac{1-\left(\gamma^{2 D-6}\right)^{N}}{1-\gamma^{2 D-6}} .
$$

By interchanging the order of the summations and the integral in Eq. (A5), the integral of $f_{n, m}^{(2)}(x)$ is given by

$$
\frac{1}{L} \int_{0}^{L} f_{n, m}^{(2)}(x) d x=\frac{\sin \phi_{n, m}}{a_{n, m}}-\frac{\sin \left(a_{n, m}+\phi_{n, m}\right)}{a_{n, m}}-\frac{\sin \left(\frac{a_{n, m}}{2}\right) \cos \left\{a_{n, m}\left(\frac{1}{2}+\frac{\tau}{L}\right)+\phi_{n, m}\right\}}{a_{n, m}} .
$$

Using $\left\langle\cos \phi_{n, m}\right\rangle=\left\langle\sin \phi_{n, m}\right\rangle=0$, the ensemble average is given by

$$
\begin{aligned}
& \left\langle\frac{A^{2}}{M L} \int_{0}^{L} \sum_{m=1}^{M} \sum_{n=0}^{N} \gamma^{2(D-3) n} f_{n, m}^{(2)}(x) d x\right\rangle= \\
& -\frac{A^{2}}{M} \sum_{m=1}^{M} \sum_{n=0}^{N} \frac{\gamma^{2(D-3) n}}{a_{n, m}}\left[\sin \left(\frac{a_{n, m}}{2}\right) \cos \left(\frac{a_{n, m}}{2}\right)+\sin \left(\frac{a_{n, m}}{2}\right) \cos \left\{a_{n, m}\left(\frac{1}{2}+\frac{\tau}{L}\right)\right\}\right] .
\end{aligned}
$$

Similarly, the integral of $f_{n, m}^{(3)}(x)$ is given by

$$
\frac{1}{L} \int_{0}^{L} f_{n, m}^{(3)}(x) d x=\frac{1}{2} \cos \left(a_{n, m} \tau\right)-\frac{\sin \left(a_{n, m} \tau+2 \phi_{n, m}\right)}{4 a_{n, m}}+\frac{\sin \left(a_{n, m}(2+\tau)+2 \phi_{n, m}\right)}{4 a_{n, m}} .
$$

Since the second and third terms vanish by ensemble averaging, we have

$$
\left\langle\frac{A^{2}}{M L} \int_{0}^{L} \sum_{m=1}^{M} \sum_{n=0}^{N} \gamma^{2(D-3) n} f_{n, m}^{(3)}(x) d x\right\rangle=\frac{A^{2}}{2 M} \sum_{m=1}^{M} \sum_{n=0}^{N} \gamma^{2(D-3) n} \cos \left(a_{n, m} \frac{\tau}{L}\right) .
$$

By combining Eqs. (A10), (A12), and (A14), we have Eq. (5).

The difference between $m / M$ and $(m+1) / M$ decreases as $M$ increases, and the summation over $m$ in Eq. (5) can be replaced with an integral. As a result, the height-height correlation function in the limit $M \rightarrow \infty$ can be expressed as

$$
R_{\infty}(\tau)=\frac{A^{2}}{2}\left[\frac{1-\left(\gamma^{2 D-6}\right)^{N}}{1-\gamma^{2 D-6}}+\sum_{n=0}^{N} \gamma^{2(D-3) n} g_{n}(\gamma, \tau)\right]
$$

where

$$
\begin{aligned}
g_{n}(\gamma, \tau) & \equiv g_{n}^{(0)}(\gamma, \tau)+g_{n}^{(1)}(\gamma)+g_{n}^{(2)}(\gamma, \tau) \\
g_{n}^{(0)}(\gamma, \tau) & =J_{0}\left(2 \pi \gamma^{n} \frac{\tau}{L}\right) \\
g_{n}^{(1)}(\gamma) & =-\frac{\pi}{2} J_{1}\left(2 \pi \gamma^{n}\right) H_{0}\left(2 \pi \gamma^{n}\right)-\frac{1}{2} J_{0}\left(2 \pi \gamma^{n}\right)\left(2-\pi H_{1}\left(2 \pi \gamma^{n}\right)\right) \\
g_{n}^{(2)}(\gamma, \tau) & =\frac{\tau}{L}{ }_{1} F_{2}\left(\frac{1}{2} ; 1, \frac{3}{2} ;-\frac{1}{4}\left(2 \pi \gamma^{n} \frac{\tau}{L}\right)^{2}\right)-\left(1+\frac{\tau}{L}\right)_{1} F_{2}\left(\frac{1}{2} ; 1, \frac{3}{2} ;-\frac{1}{4}\left(2 \pi \gamma^{n}+2 \pi \gamma^{n} \frac{\tau}{L}\right)^{2}\right) .
\end{aligned}
$$

Here, $J_{n}(x), H_{n}(x)$, and ${ }_{1} F_{2}\left(a_{1} ; b_{1}, b_{2} ; x\right)$ denote the Bessel function, the Struve function, and the generalized hypergeometric functions, respectively.
As a special case, if $\tau=0$,

$$
\langle R(0)\rangle=\frac{A^{2}}{M} \sum_{m=1}^{M} \sum_{n=0}^{N} \gamma^{2(D-3) n}\left[1-\frac{\sin a_{n, m}}{a_{n, m}}\right] .
$$


Since $2<D<3$ and $\gamma>1$, the term $\left(\gamma^{2 D-6}\right)^{N}$ vanishes in the limit of $N \rightarrow \infty$. In the limit of $M, N \rightarrow \infty$, we have

$$
\left\langle R_{c}(0)\right\rangle=A^{2}\left[\frac{1}{1-\gamma^{2 D-6}}+2 \sum_{n=0}^{\infty} \gamma^{2(D-3) n} g_{n}^{(1)}(\gamma)\right] .
$$

\section{Appendix B: Kurtosis of the distribution function for surface roughness}

Similarly with the calculation in Appendix A, the forth moment is given by

$$
m_{4}=\frac{A^{4}}{M^{2} L} \int_{0}^{L} \sum_{m_{1}=1}^{M} \sum_{n_{1}=0}^{N} \cdots \sum_{m_{4}=1}^{M} \sum_{n_{4}=0}^{N} \zeta_{n_{1}, m_{1}}(x) \zeta_{n_{2}, m_{2}}(x) \zeta_{n_{3}, m_{3}}(x) \zeta_{n_{4}, m_{4}}(x) d x
$$

The conditions that the terms in the right-hand side of Eq. (B1) can contribute to $m_{4}$ after ensemble averaging is discriminated by using the following functions:

$$
\begin{aligned}
& I_{1}=\delta_{n_{1}, n_{2}} \delta_{m_{1}, m_{2}} \delta_{n_{3}, n_{4}} \delta_{m_{3}, m_{4}}\left(1-\delta_{n_{1}, n_{3}} \delta_{m_{1}, m_{3}}\right)\left(1-\delta_{n_{1}, n_{3}} \delta_{m_{1}, M-m_{3}}\right) \\
& I_{2}=\delta_{n_{1}, n_{3}} \delta_{m_{1}, m_{3}} \delta_{n_{2}, n_{4}} \delta_{m_{2}, m_{4}}\left(1-\delta_{n_{1}, n_{2}} \delta_{m_{1}, m_{2}}\right)\left(1-\delta_{n_{1}, n_{2}} \delta_{m_{1}, M-m_{2}}\right) \\
& I_{3}=\delta_{n_{1}, n_{4}} \delta_{m_{1}, m_{4}} \delta_{n_{2}, n_{3}} \delta_{m_{2}, m_{3}}\left(1-\delta_{n_{1}, n_{2}} \delta_{m_{1}, m_{2}}\right)\left(1-\delta_{n_{1}, n_{2}} \delta_{m_{1}, M-m_{2}}\right) \\
& I_{4}=\delta_{n_{1}, n_{2}} \delta_{n_{3}, n_{4}} \delta_{m_{1}, m_{2}} \delta_{m_{3}, M-m_{1}} \delta_{m_{4}, M-m_{2}}, \\
& I_{5}=\delta_{n_{1}, n_{3}} \delta_{n_{2}, n_{4}} \delta_{m_{1}, m_{3}} \delta_{m_{2}, M-m_{1}} \delta_{m_{4}, M-m_{3}}, \\
& I_{6}=\delta_{n_{1}, n_{4}} \delta_{n_{2}, n_{3}} \delta_{m_{1}, m_{4}} \delta_{m_{2}, M-m_{1}} \delta_{m_{3}, M-m_{4}}, \\
& I_{7}=\delta_{n_{1}, n_{2}} \delta_{n_{2}, n_{3}} \delta_{n_{3}, n_{4}} \delta_{m_{1}, m_{2}} \delta_{m_{2}, m_{3}} \delta_{m_{3}, m_{4}},
\end{aligned}
$$

where $\delta_{i, j}$ denotes the Kronecker delta. If one of these functions is 1 for a combination of $n_{i}$ and $m_{i}(i=1, \ldots, 7)$, the combination contributes to $\left\langle m_{4}\right\rangle$. The total contribution from the term satisfying $I_{i}=1(i=1,2,3)$ is given by

$$
\begin{aligned}
& \left\langle m_{4}\right\rangle_{1}=3 \sum_{n=0}^{N} \sum_{m=1}^{M} \gamma^{4(D-3) n}\left(1-\frac{a_{n_{1}, m_{1}} \sin a_{n_{1}, m_{1}}-a_{n_{2}, m_{2}} \sin a_{n_{2}, m_{2}}}{a_{n_{1}, m_{1}}^{2}-a_{n_{2}, m_{2}}^{2}}\right. \\
& \left.+\frac{a_{n_{2}, m_{2}}^{3} \sin a_{n_{1}, m_{1}}-a_{n_{1}, m_{1}}^{3} \sin a_{n_{2}, m_{2}}}{a_{n_{1}, m_{1}}^{3} a_{n_{2}, m_{2}}-a_{n_{1}, m_{1}} a_{n_{2}, m_{2}}^{3}}+\frac{a_{n_{1}, m_{1}} \cos a_{n_{2}, m_{2}} \sin a_{n_{1}, m_{1}}-a_{n_{2}, m_{2}} \cos a_{n_{1}, m_{1}} \sin a_{n_{2}, m_{2}}}{a_{n_{1}, m_{1}}^{2}-a_{n_{2}, m_{2}}^{2}}\right)
\end{aligned}
$$

Similarly, the total contribution satisfying $I_{i}=1$ As a result, we obtain the averaged kurtosis: $(i=4,5,6)$ is given by

$\left\langle m_{4}\right\rangle_{2}=3 \sum_{n=0}^{N} \sum_{m=1}^{M} \gamma^{4(D-3) n}\left(\frac{3}{2}-\frac{2 \sin a_{n, m}}{a_{n, m}}+\frac{\sin 2 a_{n, m}}{4 a_{n, m}}\right)$.

The last contribution satisfying $I_{7}=1$ is given by

$$
\langle\kappa\rangle=\frac{\left\langle m_{4}\right\rangle_{1}+\left\langle m_{4}\right\rangle_{2}+\left\langle m_{4}\right\rangle_{3}}{\sigma^{2}}
$$

$\left\langle m_{4}\right\rangle_{3}=\sum_{n=0}^{N} \sum_{m=1}^{M} \gamma^{4(D-3) n}\left(\frac{9}{4}-\frac{3 \sin a_{n, m}}{a_{n, m}}+\frac{3 \sin 2 a_{n, m}}{8 a_{n, m}}\right)$

[1] J. N. Israelachvili, Intermolecular and Surface Forces, Third Edition (Academic Press, San Diego, 2011).

[2] H. B. G. Casimir, Proc. Kon. Ned. Akad. Wet. 51, 793 (1948).

[3] P. W. Milonni, The Quantum Vacuum (Academic Press, San Diego, 1994).
[4] M. Bordag, G. L. Klimchitskaya, U. Mohideen, and V. M. Mostepanenko, Advances in the Casimir Effect (Oxford University Press, New York, 2009).

[5] A. Lambrecht, A. Canaguier-Durand, R. Guérout, and S. Reynaud in Casimir Physics, Eds. D. Dalvit, P. Milonni, D. Roberts, and F. Rosa (Springer-Verlag, Heidelberg, 
2011).

[6] H. B. Chan, V. A. Aksyuk, R. N. Kleiman, D. J. Bishop, and F. Capasso, Science 291, 1941 (2001).

[7] E. Buks and M. L. Roukes, Phys. Rev. B 63, 033402 (2001).

[8] R. Decca, V. Aksyuk, and D. López in Casimir Physics, Eds. D. Dalvit, P. Milonni, D. Roberts, and F. Rosa (Springer-Verlag, Heidelberg, 2011).

[9] F. S. S. Rosa, D. A. R. Dalvit, and P. W. Milonni, Phys. Rev. Lett. 100, 183602 (2008).

[10] G. L. Klimchitskaya, A. Roy, U. Mohideen, and V. M. Mostepanenko, Phys. Rev. A 60, 3487 (1999).

[11] C. Genet, A. Lambrecht, P. Maia Neto and S. Reynaud, Europhys. Lett. 62, 484 (2003).

[12] P. J. van Zwol, V. B. Svetovoy, and G. Palasantza in Casimir Physics, Eds. D. Dalvit, P. Milonni, D. Roberts, and F. Rosa (Springer-Verlag, Heidelberg, 2011).
[13] M. V. Berry and Z. V. Lewis, Proc. R. Soc. London A 370, 459 (1980).

[14] W. Yan and K. Komvopoulos, J. Appl. Phys. 84, 3617 (1998).

[15] G. Palasantza, Phys. Rev. B 48, 14472 (1993).

[16] T. Onda, S. Shibuichi, N. Satoh, and K. Tsujii, Langmuir 12, 2125 (1996)

[17] B. Mandelbrot, Fractal Geometry of Nature (Freeman, San Francisco, 1982).

[18] M. Ausloos and D. H. Berman, Proc. R. Soc. London A 400, 331 (1985).

[19] V. B. Bezerra, G. L. Klimchitskaya, and C. Romero, Int. J. Mod. Phys. A 16, 3103 (2001).

[20] M. Bordag, G. L. Klimchitskaya, and V. M. Mostepanenko, Phys. Lett. A 200, 95 (1995). 